\title{
WSN's based Oil Well Health Monitoring and Control Using ARM9 Processor
}

\author{
B.Siri Dhatri ${ }^{1}$, Y.Chalapathi Rao ${ }^{2}$, Dr.Ch.Santhi Rani ${ }^{3}$ \\ ${ }^{1}$ M.Tech (ECE) pursuing, Bhimavaram Institute of Engg \& Tech (BIET), Pennada, Andhra Pradesh, India. \\ Email id: siri.dhatri447@gmail.com. \\ ${ }^{2} \mathrm{HOD}$ of ECE, Bhimavaram Institute of Engg \& Tech (BIET), Pennada, Andhra Pradesh, India. \\ Email id: chalu.8421@gmail.com. \\ ${ }^{3}$ Professor in ECE Department, DMSSVH College of Engg, MTM, AP, India. \\ Email id: santhirani.ece@gmail.com.
}

\begin{abstract}
The existing oil pumping system is a high power consuming process and has incapability's of CPU's structural health monitoring. Due to the environmental conditions and remote locations of oil and gas sites, it is expensive to physically visit assets for maintenance and repair. As the demand for oil and gas increases, reducing operating and maintenance costs and increasing reliability, this paper develops a sensor network based monitoring and control system, and improves the level of oil field security, enhance the security checking, and strengthen the management of digitalization and information. The system mainly consists of various sensors like temperature sensor, voltage sensor, current sensor, level sensor, $\mathrm{PH}$ sensor and gas sensor. Here we use gas sensor to detect the flammable gas which generally evolves from the oil wells. If any such detection occurs, automatically exhaust fan will switch on to pass away the particles. As like, in case if temperature level is high, cooling fan will trigger to reduce or maintain the particular temperature in the wells. With the help of current and potential transformer we can find out the fluctuations in the pumping section. If the level of oil varies from the indicated level it gives an alert message via voice recorder. To measure the level of humidity, we use $\mathrm{PH}$ sensor. All the sensors data is transmitted and monitored in PC using ARM processor. Also we can communicate this sensor data to other PC's using Zigbee technology.
\end{abstract}

\section{Keywords}

ARM Processor, Health Monitoring and Control, voice board, Wireless sensor networks (WSNs), Zigbee Module.

\section{Council for Innovative Research}

Peer Review Research Publishing System

Journal: INTERNATIONAL JOURNAL OF COMPUTERS \& TECHNOLOGY

Vol 10, No 1

editor@cirworld.com

www.cirworld.com, member.cirworld.com 


\section{INTRODUCTION}

WSN's are mainly aimed to control and monitor various applications with relatively low power consumption and large scale deployments are the main system features. The existing system consumes high power and is incapable of monitoring and controlling the operational unit [1] [2]. And it costs high to visit and control the industrial operation manually [3].

The largest volume products of the industry are fuel oil and gasoline (petrol). Previously in industries, there is no more advanced technologies to control the supply or else to monitor the pressure or humidity. The major disadvantages of existing system are

$>$ The system doesn't consider the level of fluid present in the oil well.

$>$ It doesn't check the temperature conditions of the gas source.

$>$ It doesn't identify the flow of gas.

$>$ No more accuracy

The security management system for oil field in this paper adopted wireless communication technology and embedded system. This paper discusses the hardware and software of wireless communication in the system mainly. It improves, enhances and strengthens the oil field security [4] with the usage of various sensors which continuously senses and monitors the system [5]. It is mainly aimed at the environment for lack of power and designs emergency remote security monitoring and control system based on ARM processor [6]. Uses advanced RISC machine processor embedded with the real time systems to monitor and control oil industries [7].

The method could solve problems with highly efficient and rapid, also could reduce the loss of country effectively. So it has the important significance and social and economic benefits for the dependability and the controllability of remote communication [8].

Minimize harmful effects

$>$ Technology cost modules for manufacturers.

$>$ Highly efficient.

$>$ More trusted one and a better revolution for industries.

$>$ Ease of maintenance.

The monitoring and controlling section of this system mainly consists of microcontrollers AT89S52 and LPC3130 and the sensors data is communicated via Zigbee

\subsection{Function of AT89S52}

The AT89S52 is a low-power, high-performance CMOS 8-bit microcontroller with 8K bytes of in-system programmable flash memory. The device is manufactured using Atmel's high-density non volatile memory technology and is compatible with the industry-standard 80C51 instruction set and pin out. The on-chip Flash allows the program memory to be reprogrammed in-system or by a conventional non volatile memory programmer. By combining a versatile 8-bit CPU with in-system programmable Flash on a monolithic chip, the Atmel AT89S52 become a powerful microcontroller which provides a highly-flexible and cost-effective solution to many embedded control applications.

The AT89S52 provide the following standard features:

$8 \mathrm{~K}$ bytes of flash, 256 bytes of RAM, 32 I/O lines, Watchdog timer, two data pointers, three 16-bit Timers/counters, a sixvector two level interrupt architecture, a full duplex serial port, on-chip oscillator, and clock circuitry. In addition, the AT89S52 is designed with static logic for operation down to zero frequency and supports two software selectable power saving modes. The Idle mode stops the CPU while allowing the RAM, timer/counters, serial port, and interrupt system to continue functioning. The power down mode saves the RAM contents but freezes the oscillator, disabling all other chip functions until the next interrupt or hardware reset.

\subsection{Function of LPC3130}

LPC is a family of microcontroller ICs by NXP semiconductors (formerly Philip semiconductors).The LPC chips are based on the 32-bit RISC ARM cores from ARM Holdings, such as Cortex-M4F, Cortex-M3, Cortex-M0+, Cortex-M0, ARM9 and ARM7 cores.

The NXP LPC 3130/3131 combine an $180 \mathrm{MHz}$ ARM926EJ-S CPU core, high speed USB 2.0 On-The-Go (OTG), up to 192 KB SRAM, NAND flash controller, flexible external bus interface, four channel 10 bit ADC, and a myriad of serial and parallel interfaces in a single chip targeted at consumer, industrial, medical and communication markets. To optimize system power consumption, the LPC3130 have multiple power domains and a very flexible Clock Generation Unit (CGU) that provides dynamic clock gating and scaling. 
The processor embedded in the LPC3130 is the ARM926EJ-S. It is the member of the ARM9 family generalpurpose microprocessors. It is intended for multi-tasking applications where fully memory management, high performance, and low-power are important.

The main features of this module are as follows.

$>$ Uses five-stage pipeline consisting of fetch, decode, execute, memory, and write stages.

$>$ Contains an AMBA BIU for both data accesses and instruction fetches.

$>16 \mathrm{~KB}$ instruction and $16 \mathrm{~KB}$ data separate cache memories with an 8 word line length.

$>$ Supports ARM debug architecture.

$>$ Supports dynamic clock gating for power reduction.

$>$ Supports coprocessor interfacing.

The ARM926EJ-S supports the connection of on-chip coprocessors to the ARM9EJ-S core through an external coprocessor interface. Coprocessors determine the instructions that they have to execute by using a pipeline follower in the coprocessor. As each instruction arrives from memory, it enters both the ARM9EJ-S pipeline and the coprocessor pipeline. figure 1.

The Coprocessor interface of ARM926EJ-S processor through CP15 system configuration coprocessor is shown in

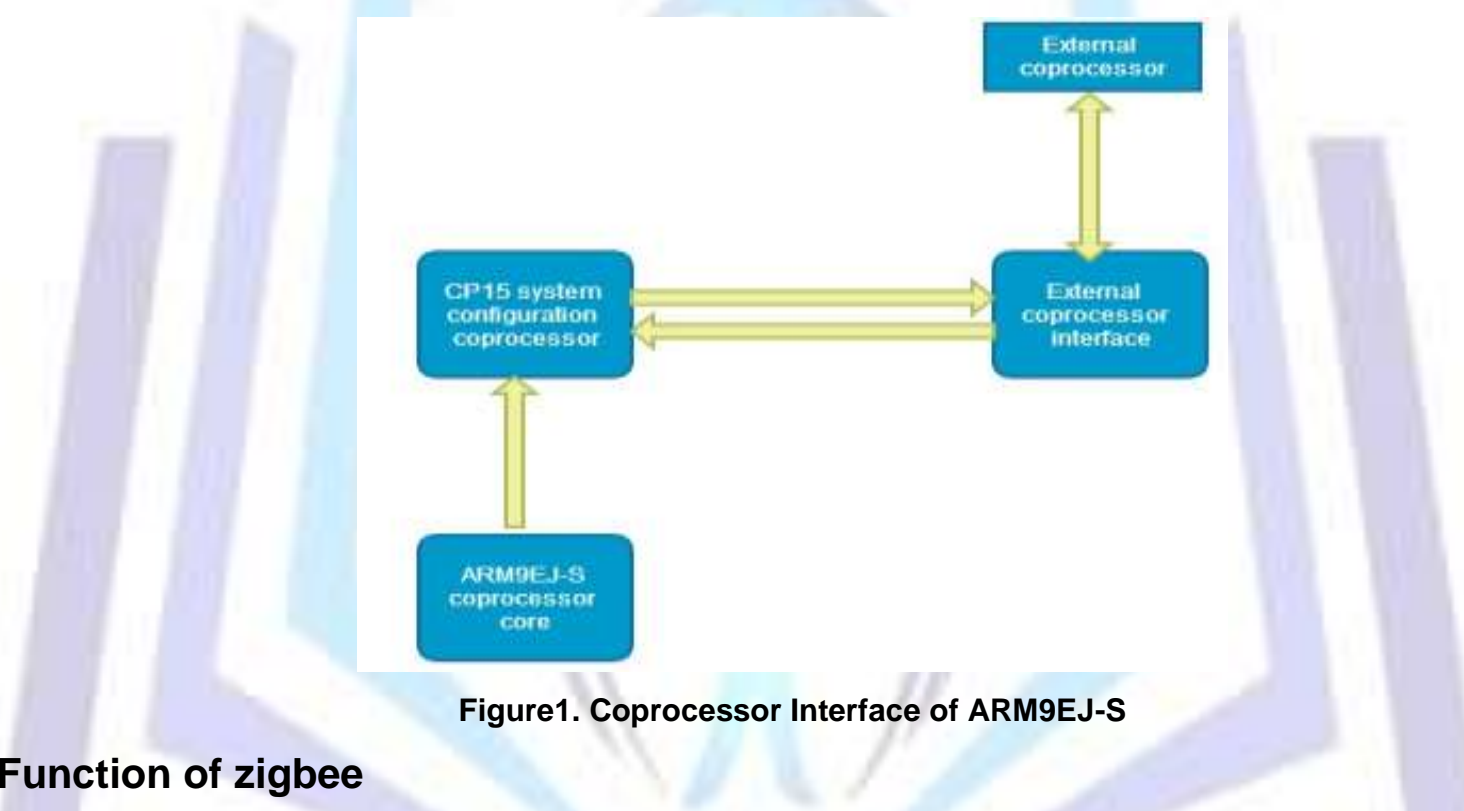

Zigbee is a specification for a suite of high level communication protocols used to create personal area networks built from small, low-power digital radios. Zigbee is based on an IEEE 802 standard. Though low-powered, Zigbee devices often transmit data over longer distances through intermediate devices to reach more distant ones, creating a mesh network: i.e., a network with no centralized control or high-power transmitter/receiver able to reach all of the networked devices. The decentralized nature of such wireless ad-hoc networks make them suitable for applications where a central node can't be relied upon.

Zigbee is used in applications that require a low data rate, long battery life, and secure networking. Zigbee has a defined rate of $250 \mathrm{Kbits} / \mathrm{sec}$, best suited for periodic or intermittent data or a single signal transmission from a sensor or input device. Applications include wireless light switches, electrical meters with in-home-displays, traffic management systems, and other consumer and industrial equipment that require short-range wireless transfer of data at relatively low rates. The technology defined by the Zigbee specification is intended to be simpler and less expensive than other WPANs, such as Bluetooth or Wi-Fi.

\section{SYSTEM STRUCTURE}

The system structure mainly consists of Transmitter Section and Receiver Section which are shown in figure 1 and figure 2 respectively.

Transmitter section mainly consists of six sensor networks in this system which senses various effects near the oil wells. Various sensors used here are Temperature, gas, $\mathrm{PH}$, Level, Voltage and Current Sensors. As the output through these sensors is a physical quantity, they are connected to ADC (Analog to Digital Converter) to convert this analog information to digital format and then this digital information is monitored through AT89S52 microcontroller. 
The controlling section of this system is of great interest. The entire sensor's data are stored in the processor memory and continuously monitored. If any of the sensors exceeds its threshold level, it indicates the workers through the voice board which have a connection to the microcontroller. Initially the voice is recorded in the voice board and when a particular sensor crosses the limit, it announces that the sensor level is high. Also we can automatically control the environment of oil wells if any sensor level is high. Here two external devices are connected to the system through the relay. They are Exhaust fan and cooling fan. When the temperature is high, then the switch connected to cooling fan is ON and when $\mathrm{CO}_{2}$ gas level is high, then the switch connected to exhaust fan is $\mathrm{ON}$.

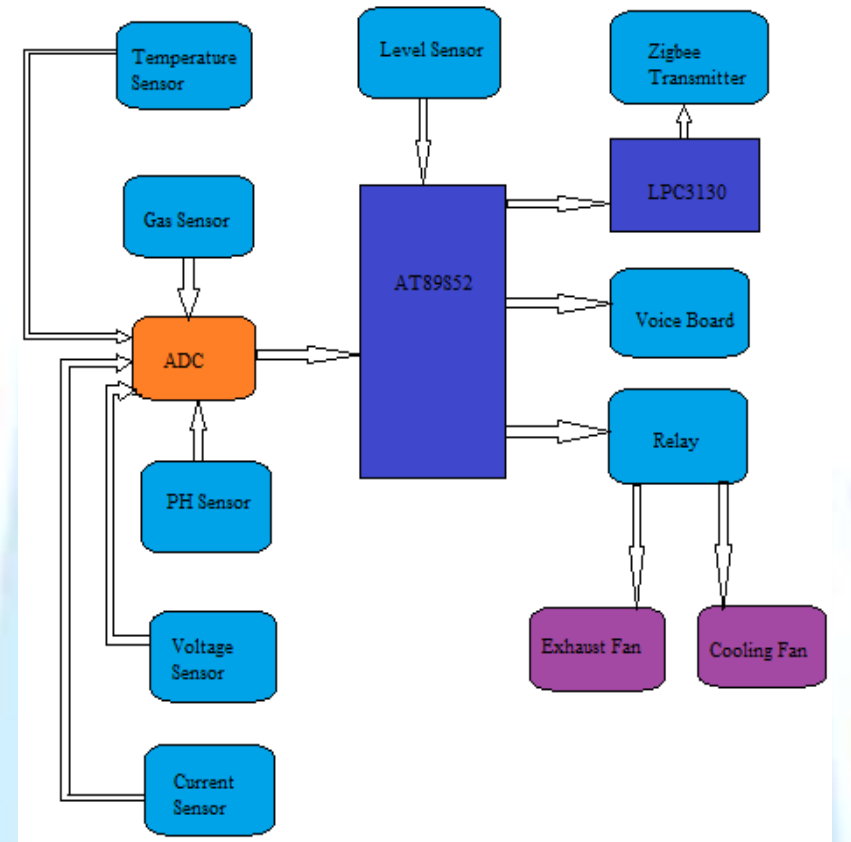

Figure 2: Block diagram: Transmitter Section

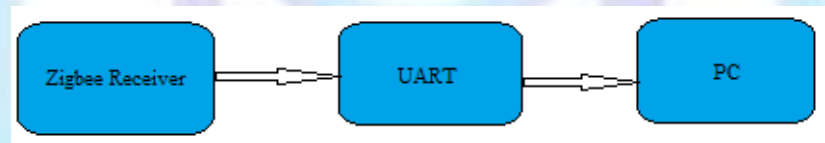

Figure 3: Block Diagram: Receiver Section

Not only the sensor's level is indicated to the workers at working environment through voice board, we can also transmit this data to other PC's using Zigbee module. Zigbee transmitter is connected to LPC3130 controller. It continuously checks out the sensor data and sends to the Zigbee receiver. This Zigbee receiver is connected to the PC through UART as the voltage levels are not equal. We can communicate the sensor's data to other PC's through Zigbee up to 200 meters long.

\section{HARDWARE DEPLOYEMENT}

\subsection{Function of Current sensor} to it.

A Current sensor is a device that detects electrical current $(A C$ or $D C)$ in a wire, and generates a signal proportional

The sensed current and output signal can be:

- $A C$ current input,

- Analog output, which duplicates the wave shape of the sensed current

- Unipolar output, which is proportional to the average or RMS value of the sensed current.

- DC current input,

○ Unipolar, with a unipolar output, which duplicates the wave shape of the sensed current.

○ Bipolar output, which duplicates the wave shape of the sensed current. 
○ Digital output, which switches when the sensed current exceeds a certain threshold.

\subsection{Function of Voltage sensor}

The interface of various sensors to a controller like the BrainStem GP 1.0 module typically involves either conditioning or converting voltage levels in the range the controller requires. Many systems use $A / D$ converters to make the sensor value relevant in a program or data logging configuration. These converters have a fixed range of voltages they can convert from with $0-5$ volts being by far the most common.

Sensors often create voltages in different ranges than those required by the controllers they are being interfaced to which requires the conversion of one voltage to another. This conversion often breaks down into a combination of one or more of three types- amplification, dividing and shifting.

\subsection{Function of Temperature sensor}

The measurement of temperature is one of the fundamental requirements for environmental control, as well as certain chemical, electrical and mechanical controls. Many different types of temperature sensors are commercially available, and the type of temperature sensor that will be used in particular application will depend on several factors. For example, cost, space constraints, durability, and accuracy of the temperature sensor are all considerations that typically need to be taken into account

In this paper we use LM35 as a temperature sensor. LM35 is a precision IC temperature sensor with its output proportional to the temperature (in ${ }^{0} \mathrm{C}$ ). The sensor circuitry is sealed and therefore it is not subjected to oxidation and other processes. With LM35, temperature can be measured more accurately than with a thermistor. It also possesses low self heating and does not cause more than $0.1^{\circ} \mathrm{C}$ temperature rise in still air.

The operating temperature range is from $-55^{\circ} \mathrm{C}$ to $150^{\circ} \mathrm{C}$. The output voltage varies by $10 \mathrm{mV}$ in response to every ${ }^{0} \mathrm{C}$ rise/fall in ambient temperature i.e., its scale factor is $0.01 \mathrm{~V} /{ }^{0} \mathrm{C}$.

\subsection{Function of Level sensor}

Level sensors detect the level of substances that flow, including liquids, slurries, granular materials, and powders. All such substances flow to become essentially level in their containers (or other physical boundaries) because of gravity. The substance to be measured can be inside a container or can be in its natural form (e.g. a river or a lake).

The level measurement can be either continuous or point values. Continuous level sensors measure level within a specified range and determine the exact amount of substance in a certain place, while point-level sensors only indicate whether the substance is above or below the sensing point. Generally the latter detect levels that are excessively high or low.

Also important are the application constraints: price, accuracy, appearance, response rate, ease of calibration or programming, physical size and mounting of the instrument, monitoring and control of continuous or discrete (point) levels.

\subsection{Function of $\mathrm{PH}$ sensor}

The most common $\mathrm{PH}$ sensor is the glass electrode. It is used in many industry applications and in a wide variety of fields. The glass-electrode method has a high reproducibility, and it can measure $\mathrm{PH}$ of various solutions.

Here we are using an electro chemical $\mathrm{PH}$ sensor. A PH electrode is a potentiometric or electrochemical sensor that has a voltage output. A potentiometric sensor consists of two electrochemical cells or electrodes.

- The glass electrode, sometimes called the measuring electrode or active electrode

- And the reference electrode.

The electric potential created between the glass electrode, and the reference electrode is a function of the $\mathrm{PH}$ value of the measured solution. So once the potential difference has been measured, we can calculate the $\mathrm{PH}$ value.

\subsection{Function of Gas sensor}

Gas detectors and gas sensors interact with a gas to initiate the measurement of its concentration. The gas detector of gas sensor then provides output to a gas instrument to display the measurements. Common gases measured by gas detectors and gas sensors include ammonia, aerosols, arsine, bromine, carbon dioxide, carbon monoxide, chlorine, chlorine dioxide etc.

In this paper we use MQ7 type gas sensor. A typical Sensitivity characteristic of MQ-7 gas sensor [9] is shown below.

They are used in gas leakage detecting equipments in family and industry, are suitable for detecting of LPG, isobutane, propane, LNG, avoid the noise of alcohol and cooking fumes and cigarette smoke. 


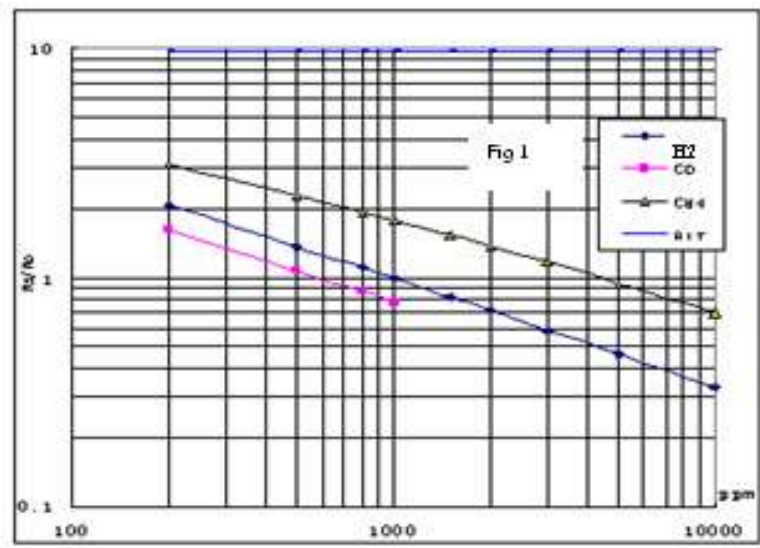

Figure 4: Typical Sensitivity Characteristics of MQ-7

\subsection{Function of $A D C$}

In this paper we are using ADC0808 converters to convert the analog data coming from various sensors to digital data The ADC0808 data acquisition component is a monolithic CMOS device with an 8-bit analog-to-digital converter, 8channel multiplexer and microprocessor compatible control logic. The 8-bit A/D converter uses successive approximation as the conversion technique. The converter features a high impedance chopper stabilized comparator, a 256R voltage divider with analog switch tree a successive approximation register. The 8-channel multiplexer can directly access any of 8-single-ended analog signals. The device eliminates the need for external zero and full-scale adjustments. Easy interfacing to microprocessors is provided by the latched and decoded multiplexer address inputs and latched latched TTL TRI-STATE outputs. The design of the ADC0808, AD0809 has been optimized by incorporating the most desirable aspects of several A/D conversion techniques. The ADC0808, ADC0809 offers high speed, high accuracy, minimal temperature dependence, excellent long-term accuracy and repeatability and consumes minimal power. These features make this device ideally suited to applications from process and machine control to consumer and automotive applications.

\subsection{Function of Voice board}

The APR9600 experimental boards is an assembled PCB board consisting of an APR9600 IC, an electric microphone, support components and necessary switches to allow users to explore all functions of the APR9600 chip. The oscillation resistor is chosen so that the total recording period is 60 seconds with a sampling rate of $4.2 \mathrm{kHz} \mathrm{V}$.

\subsection{Function of Relay}

A relay is an electrically operated switch. Current flowing through the coil of the relay creates a magnetic field which attracts a lever and changes the switch contacts. The coil current can be ON or OFF. So relays have two switch positions and they are double throw (changeover) switches.

Relays allow one circuit to switch a second circuit which can be completely separate from the first. For example, a low voltage battery circuit can use a relay to switch a 230V AC mains circuit. There is no electrical connection inside the relay between the two circuits; the link is magnetic and mechanical.

The coil of the relay passes a relatively large current from low voltages. Most IC's (chips) cannot provide this current and a transistor is usually used to amplify the small IC current to the larger value required for the relay coil. The maximum output current for the popular 555 timer IC is $200 \mathrm{~mA}$. So these devices can supply relay coils directly without amplification.

Relays are usually SPDT or DPDT but they can have many more sets of switch contacts. Most relays are designed for PCB mounting but we can solder wires directly to the pins providing to take care to avoid melting the plastic case of the relay.

\subsection{Function of UART}

A Universal Asynchronous Receiver/Transmitter is a type of "Asynchronous Receiver/Transmitter", a piece of computer hardware that translates between parallel and serial forms. UARTs are commonly used in conjunction with other communication standards such as EIA RS-232.

A UART is usually an individual integrated circuit used for serial communication over a computer or peripheral serial port. UARTs are now commonly included in microcontrollers. A dual UART or DUART combines two UARTs into a single chip.

The UART controller is the key component of the serial communication subsystem of a computer. It takes bytes of data and transmits individual bits in a sequential fashion. At the destination, a second UART re-assembles the bits into complete bytes. Each UART contains a shift register which is the fundamental method of conversion between serial and parallel forms. 


\section{APPLICATIONS}

Wireless sensor networks are used in wide range of applications, mainly in monitoring and control of various industries [10] [11]. Some of them include oil and gas, cold chain and machine health monitoring, in pipelining etc [12]

\subsection{Oil and Gas industries}

Oil and gas manufacturing is one industry looking towards wireless sensor networks. New wireless mesh networking topologies are one factor that drives new levels of reliability. In a mesh network topology, each node has at least two parent nodes with which it can communicate. Even if an individual link becomes inoperable, a node still has a communication path available. This redundant routing ensures resiliency in case of offline nodes or broken links.

\subsection{Pipelining}

The ability to monitor flow and pressure build-ups of the multiple phase mixture (oil, gases and brine) through a pipeline in real-time is critical for the safe and efficient transport of fluids. Because pipelines may span many kilometres along the sea bed, normal maintenance procedures at a depth of up to 10,000 feet are costly both in time and resources. Failures due to corrosion could cause release of oil and gas, which negatively impacts the environment, causes loss of production and presents a significant safety hazard.

The temperature fluid in pipelines will typically differ from that of the atmospheric temperature and will therefore have its own temperature signature which will be proportionate to the flow of the fluid. In the event of a leak in the pipeline, the flow downstream of the leak will be less than before the leak, and therefore, there will be different temperature profiles before and after the leak location.

\section{RESULT ANALYSIS}

The transmitter section consists of microcontroller AT89S52, LPC3130, various sensors, voice board, relay circuit to switch exhaust fan and cooling fan and a Zigbee transmitter. The receiver section consists of a Zigbee receiver and this module is connected to a PC present at some distance through UART for communication.

In oil and gas industries, there are drastic changes in the temperature. It may be up to some 100 's of ${ }^{0} \mathrm{C}$. Here a prototype of temperature sensor LM 35 is used which shows the values of temperature variations ranging from 0 to $270^{\circ} \mathrm{C}$. Similarly a prototype of gas sensor MQ-7 is used in this paper which mainly detects the release of Carbon dioxide gas. Level sensor indicates whether oil wells are fully filled or not. If it is empty, then it indicates 'LOW', if it is full, it indicates 'HIGH'. Voltage and Current sensors continuously check the levels in the industry and automatically switches off the supply to the particular module. If any unwanted fluids are released in the industries, they can be sensed by $\mathrm{PH}$ sensor. If any such fluids are detected, PH sensor level shows high; generally it indicates a value of 255 .

All these sensors nodes are continuously monitored by the microcontroller AT89S52. This processor is interfaced with the LPC3130 ARM926EJ-S processor. The controlling of sensor nodes and voice board and Zigbee communication is done by LPC3130 controller. The voice board continuously announces the sensor levels and also this sensors data is communicated to other PC's using Zigbee module. The result analysis of this paper is shown below.

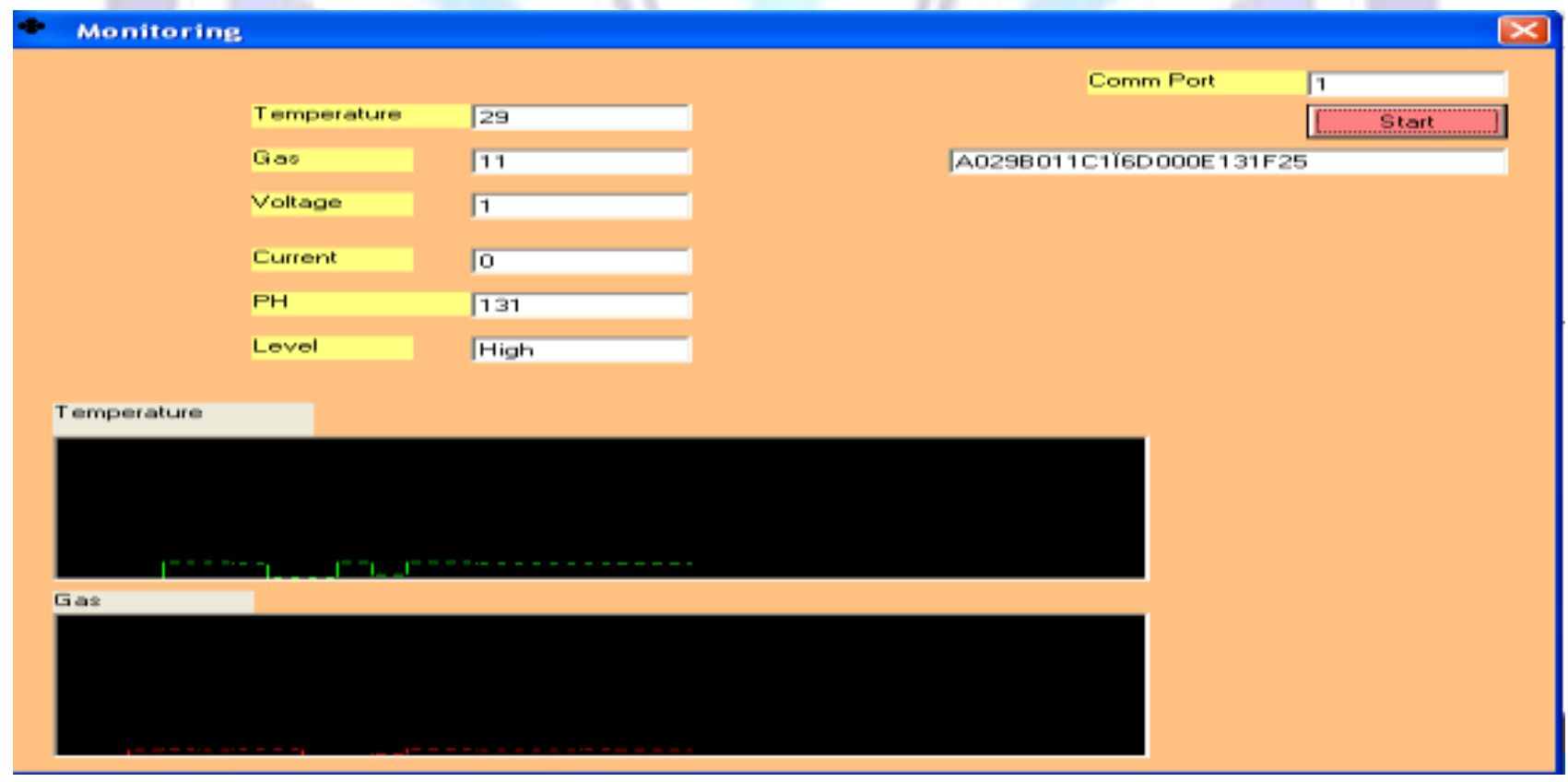

Figure 5: Expected result of the system 
The figure represents the sensors data when the temperature is at normal condition, no voltage and current fluctuations occur in the industry, when unwanted fluids are released i.e., when $\mathrm{PH}$ is high, when the level of oil well is high.

\section{CONCLUSION}

The hardware and software design of an embedded monitoring system for real time applications is presented in this paper. Vibration signals have been analyzed to detect the mechanical faults and necessary steps are taken to reduce the faults. The implementations of analysis technique in time and frequency domain are given. The proposed system oil well health monitoring and control based on WSNs is verified with different levels of severity.

\section{REFERENCES}

[1] Donglin Wang, Member IEEE, Renlun He, Jiangqiu Han, Michel Fattoucho and Fadhel M. Ghonnouchi, Fellow, IEEE, "Sensor Network based Oil well Health Monitoring and Intelligent Control", IEEE Sensors Journal, vol 12, No. 5, May 2012.

[2] V. Viknesh Velavan and R.Chandralekha, "Oil Well Monitoring and Control based on Wireless Sensor Networks Using ARM", International Journal of Electronics and Computer Science Engineering, Volume 1, Number 4.

[3] Oil and Gas Condition Monitoring, National Instruments, ni.com.

[4] Jiang Wei,"Security Management System for Oilfield Based On Embedded Wireless Communication", 2009 International Conference on Network Security Wireless Communications and Trusted Computing. Issue Date: 25-26 April 2009, On page(s): 604 - 606, INSPEC Accession Number: 10627363.

[5] C.Rojiha, "Sensor Network Based Automatic Control System for Oil Pumping Unit Management", International Journal of Scientific and Research Publications, Volume 3, Issue 3, March 2013

[6] Shaocheng Song, "Design of emergency remote security monitoring and control system based on ARM", In proceeding of 2nd International IEEE conference on Consumer Electronics, Communications and Networks, April 2012.

[7] Xiao Liang, En Li, Zizi Liang, "Intelligent Monitoring System based on the Embedded Technology", Proceeding of the 2007 IEEE International Conference on TuesE04 Networking ,Sensing and Control, London, UK, 15-17 April.

[8] Ganesh V. Padole, Sandip N. Kamble, "Embedded Wireless based Communication in Oilfield and Providing Security System", International Journal Communication and Network Security (IJCNS), vol I, Issue II, 2011.

[9] MQ-7 Semiconductor Sensor for Carbon Monoxide, Henan Hanwei Electronics Co., Ltd, www.hwsensor.com.

[10] Sudararajan, V.; A. Redfern; M. Schneider; and P. Wright (2005). "Wireless Sensor Networks for Machinery Monitoring," ASME International Mechanical Engineering Congress and Exposition.

[11] Wright, P.; D. Dornfeld; R. Hillaire; and N. Ota (2006). "Tool Temperature Measurement and its Integration within a Manufacturing System." Transactions of NAMRI/SME, Vol. 34, pp. 63-70.

[12] Jagannath, V.M.D and B.Raman (2007). "WiBeam: Wireless Bearing Monitoring System" communication systems software and Middleware, COMSWARE 2007, $2^{\text {nd }}$ International conference. 